Document downloaded from:

http://hdl.handle.net/10251/162573

This paper must be cited as:

Coll, C.; Sánchez, E. (2019). Quarantine in an epidemic model with seasonality. Revista de la Real Academia de Ciencias Exactas Físicas y Naturales Serie A Matemáticas. 114(1):112. https://doi.org/10.1007/s13398-019-00753-x

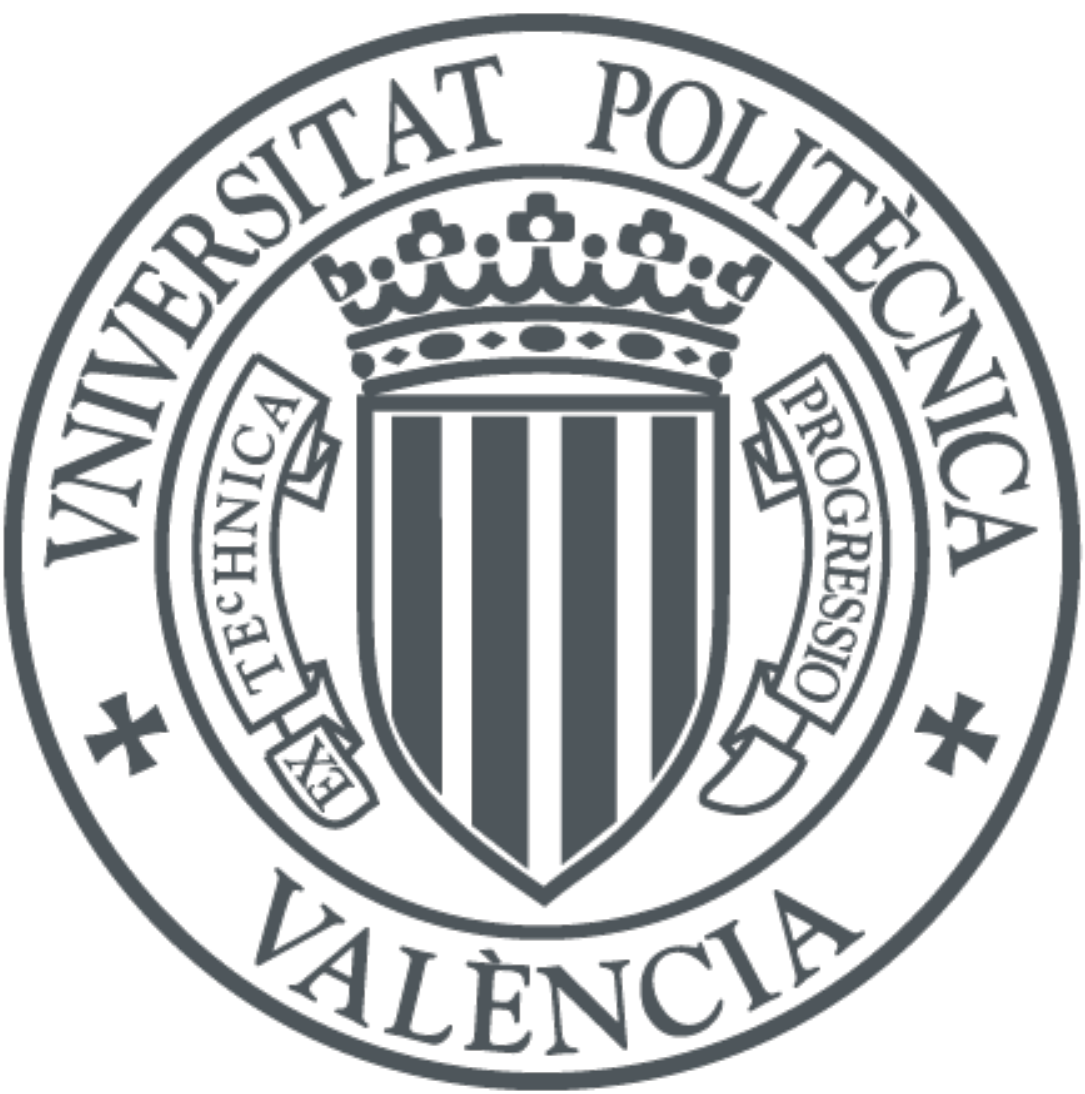

The final publication is available at

https://doi.org/10.1007/s13398-019-00753-x

Copyright Springer-Verlag

Additional Information 


\title{
Quarantine in an epidemic model with seasonality
}

\author{
Carmen Coll · Elena Sánchez
}

Received: date / Accepted: date

\begin{abstract}
In this work we focus on the periodicity of recurrent epidemic patterns and propose a periodic model that includes quarantine as a control strategy. So, we consider that susceptible individuals can be quarantined $(Q)$ and then return to the recovered class once it is determined that they are not infected. We use the basic reproductive number $\mathcal{R}_{0}$ to analyze the spread of the disease. To study the effectiveness of quarantine in the spread of the disease, a new parameter $\mathcal{R}_{q}$ is introduced. This is defined as the average number of new infections that a case generates, in a totally susceptible population when quarantine is applied to individuals during the time they are infectious. The value of $\mathcal{R}_{q}$ will be closely related to both the intensity of the intervention and the severity of the epidemic in the absence of such intervention. The behavior in absence of control will be determined by the value of the basic reproductive number $\mathcal{R}_{0}$.
\end{abstract}

Keywords Quarantine · periodic model · epidemic model · basic reproductive number

Mathematics Subject Classification (2010) MSC 92D30 - MSC 93C55 . MSC 93D20

C.Coll

Institut Universitari de Matemàtica Multidisciplinar, Universitat Politècnica de València, Valencia Spain

Tel.: +34-96-3877665

E-mail: mccoll@mat.upv.es

E.Sánchez

Institut Universitari de Matemàtica Multidisciplinar, Universitat Politècnica de València, Valencia Spain

Tel.: +34-96-3877665

E-mail: esanchezj@mat.upv.es 


\section{Introduction}

Mathematical models have been used to describe recurrent epidemic patterns in a given population and as tools to design control strategies and to predict the effect of such interventions on mitigating future epidemics or pandemics. The SIR is the most used classic model. In this model the population is partitioned into three classes with the susceptible individuals to the disease, the infected individuals and recovered individuals. Many epidemics exhibit seasonality. Some examples appear when temporal patterns in human activity are considered, like the start of the school year, or the effects of weather on the population. Examples of seasonal diseases are the chidlhood infectious diseases such as influenza, measles, varicella or mumps or infectious diseases in animals such as swine flu. In Coburn et al.(2009) it can find a good review of models and control techniques for swine flu, in Stone et al. (2007) it can find a good discussion on seasonal models of recurrent epidemics and in Cao and Zhou (2013) is defined the basic reproduction number for epidemic models with periodic parameters.

Vaccination is among the most frequent control techniques, however, this is very costly, even in rich countries, to achieve an acceptable level of vaccination to control the disease. Therefore, it is interesting to analyze, in seasonal models, what effect other control interventions produce, such as the closure of schools, the quarantine of infected persons or the imposition of travel restrictions. In recent years, the effect of these types of interventions in the case of influenza has been studied by several authors (Cauchemez et al.(2008) ; Epstein et al. (2007) ; Fumanelli et al. (2016) ).

In the last decades, interest in developing models that predict the level of effectiveness of strategies such as isolation or quarantine to stop the spread of an infectious disease has increased. In Hethcote et al. (2002), the effects of quarantine in six endemic models for infectious diseases has been studied with the peculiarity that each of these models has an analogous model with an exponential demographic structure. Castillo-Chavez et al. (2003) presents a summary of how mathematical models can help determine the effectiveness of actions such as isolation or quarantine in the control of infectious diseases. Muyabi et al. (2010) analyzes the economic impact associated with the joint implementation of quarantines and isolation strategies. For this analysis, the quarantine process is modeled after eliminating a proportion of individuals per unit of time in all compartments. In particular, this has the effect of reducing the number of susceptible individuals in the population and, therefore, the incidence. Most of the previous results are based on models where their parameters are constant. Unfortunately, seasonality causes the process to be executed periodically every units of time. Therefore, the periodicity assumption leads to consider models with variable coefficients. The main contribution of our model is how the periodicity of the parameters has been introduced in the dynamic evolution of the model.

The notation and the basic concepts that will use throughout the work is followed. A matrix $M=\left(m_{i j}\right)$ is non-negative if $m_{i j} \geq 0$ for all $i, j$, and it is 
denoted by $M \geq O$. The spectrum of $M$ and its spectral radius are denoted by $\sigma(M)$ and $\rho(M)$, respectively, and, $M$ is stable if $\rho(M)<1$ (see Berman and Plemmons (1994) ). Moreover, the expression $\operatorname{col}\left(X_{i}\right)_{i=1}^{l}$ denotes a column block matrix whose blocks are $X_{i}, i=1, \ldots, l$. Finally, recall that a circulant matrix $A_{c}$ is a matrix where the first row of $A_{c}$ is $\left(a_{0}, a_{1}, \cdots, a_{i}, \cdots, a_{n-1}\right)$ and its $(i+1)$-th row is obtained by giving its $i$-th row a right circular shift by one positions (Davis (1979)).

Consider an invariant discrete-time system

$$
x(t+1)=A x(t), \quad A=T+F, \quad k \geq 0,
$$

where the vector $x(t)$ represents the infected individuals at time $t$ and $T \geq O$ and $F \geq O$ represent the transition matrix and the infection matrix, respectively and we assume that the initial system without new infections is asymptotically stable $\rho(T)<1$.

The basic reproduction number, $\mathcal{R}_{0}$, quantifies the severity of the disease. $\mathcal{R}_{0}$ measures the average number of secondary cases of infection that one infected individual generates, in a susceptible population, during the time which the individual is infectious. One way of calculating $\mathcal{R}_{0}$ is using the next generation method where $\mathcal{R}_{0}$ is the spectral radius of the next generation matrix. So, this indicator is defined by $\mathcal{R}_{0}=\rho\left(F(I-T)^{-1}\right)$, Allen and Driessche (2008). It is known (Castillo-Chavez et al. (2002) ; Li and Schneiser (2002); Cao and Zhou (2013)) that if $\mathcal{R}_{0}<1$ then the system (1) is asymptotically stable, that is, $\rho(A)=\rho(T+F)<1$ and the disease disappears. Moreover, if $\mathcal{R}_{0} \geq 1$, the system (1) is not asymptotically stable, $\rho(A)=\rho(T+F) \geq 1$, in this case the epidemic can possibly establish in the population and increase rapidly.

In this work, we focus on the periodicity of a seasonality disease. This seasonality leads to the periodicity of the coefficient matrices of the model, defined in terms of its epidemiological parameters. In this case the process is represented by an $P$-periodic system given by

$$
x(t+1)=A(t) x(t), \quad A(t)=T(t)+F(t), \quad t \geq 0,
$$

where the matrices are $P$-periodic matrices. In the next formulations the term invariant is used to indicate, that unlike the initial model, the matrices of the coefficients are constant.

Generally, the following two invariant formulations can be associated to this $P$-periodic system:

- PIS: A collection of $P$ invariant systems (see Meyer and Burrus (1975)), given by

$$
x_{s}(t+1)=A_{s} x_{s}(t), \quad A_{s}=T_{s}+B_{s} F_{s}, \quad t \geq 0,
$$

where

$$
\begin{aligned}
& x_{s}(t)=x(t P+s), B_{s}=\operatorname{row}\left(\Phi_{T}(s+N, s+1+j)\right)_{j=0}^{P-1} \\
& T_{s}=\Phi_{T}(s+P, s) \quad F_{s}=\operatorname{col}\left(F(s+j) \Phi_{A}(s+j, s)\right)_{j=0}^{P-1},
\end{aligned}
$$




$$
\Phi_{T}\left(s, s_{0}\right)=\left\{\begin{array}{l}
\prod_{i=s_{0}}^{s-1} T\left(s_{0}+s-1-i\right), s>s_{0} \\
I_{n}, s=s_{0}
\end{array}\right.
$$

for $s=0,1, \ldots, P-1$.

- ICAS: An invariant system called Invariant Cyclically Augmented System, that recollect all the information of a period (see Park and Verriest (1989)), which state is defined by $z(t)=M_{n}^{t-1} \widehat{x}(t)$, with $\widehat{x}(t)=$ $\operatorname{col}(x(t+j))_{j=0}^{N-1}$, and $M_{j}=\left(\begin{array}{cc}O & I_{(P-1) j} \\ I_{j} & O\end{array}\right)$, with $j>0$ and we consider the matrix $M_{e}$ defined from an $P$-periodic collection of matrices $\{M(0), M(1), \cdots, M(P-1)\}$ as

$$
M_{e}=\left(\begin{array}{cccc}
O & M(0) \\
\operatorname{diag}(M(1) & \cdots & M(P-1)) & O
\end{array}\right)
$$

This ICAS system is given by

$$
z(t+1)=A_{e} z(t), \quad A_{e}=T_{e}+F_{e}, \quad t \geq 0
$$

where $T_{e}$ and $F_{e}$ are defined using the its corresponding $P$-periodic collection of matrices.

It is known that the $P$-periodic system is asymptotically stable if and only if $\rho\left(A_{s}\right)<1$ or equivalently $\rho\left(A_{e}^{P}\right)<1$, since $A_{e}^{P}=\operatorname{diag}\left(A_{1} A_{2} \cdots A_{P-1} A_{0}\right)$.

The concept of basic reproduction number for $P$-periodic system is given using these invariant formulations. Thus, in Bacaër and Ait (2012), it is defined using the basic reproduction number of its ICAS formulation, that is, $\mathcal{R}_{0}^{e}=$ $\rho\left(F_{e}\left(I-T_{e}\right)^{-1}\right)$ and in Cushing and Ackleh (2012) it is defined using its PIS formulation, that is, $\mathcal{R}_{0}^{s}=\rho\left(B_{s} F_{s}\left(I-T_{s}\right)^{-1}\right), s=0,1, \ldots P-1$. Recently, in Cantó et al. (2014) is introduced a collection of $s$-basic reproduction numbers to analyze the eradication of a disease in a periodic seasonal process.

The rest of the paper is organized as follows. In Section 2, an epidemic model is presented in a discrete time that shows the state variables and the epidemiological parameters involved in the process, and how they relate to each other. In Section 3, the effects of the quarantine strategy in the initial model are analyzed and the influence of the quarantine on the stability of the model is investigated taking into account the parameters of the process. Finally, we give some conclusions in Section 4.

\section{Mathematical model}

Initially, we consider a SIR model consisting of three compartments: Susceptible $(S)$, Infected $(I)$, and Immune or Recovered $(R)$. The process with which the disease spreads is as follows: people who become infected come from class $S$ and go on to class $I$ depending on the virulence or prevalence of the infection, 
an infected individual can continue to be infected, recover or die. It is assumed that, after recovery, individuals do not take part in the transmission process. Quarantine is one of the strategies used to control the spreading of infectious diseases. This strategy consists of isolating those members of the population who are suspected of being infected but have not yet been diagnosed. This measure is taken to prevent the spread of diseases and it is usually effective in many diseases with an incubation period ( or a latent period) in which infected individuals can not present the onset of symptomatic disease (or do not transmit the disease to susceptible individuals). There is a wide spectrum of diseases that can be asymptomatic during a more or less prolonged period of time, some examples are ebola, malaria and influenza. So, the Quarantine $(Q)$ compartment is considered. Thus, we denote by $S(t), I(t), Q(t)$ and $R(t)$ the density of individuals at time $t$ of each compartment. In addition, we assume that the population $N(t)$ approaches a limiting population size $N$. The theory of asymptotically autonomous systems implies that if $N(t)$ has a constant limit $N$ then the system is equivalent to the system in which $N(t)$ is replaced by this limit. This applies here and it assumes that the population has constant size $N$.

The death removal rate at time $t$ is denoted by $\mu(t)$ and it determines the inflow of elements into the susceptible class. Parameters $0<p<1,0<q<1$, $0<s<1$ and $0<r<1$ are the survival rate of the $S(t), I(t), Q(t)$ and $R(t)$ individuals, finally, $\alpha(t)$ and $\gamma(t)$ are the transmission rate and the recovery rate per day respectively. The parameter $\omega(t)$ describes the rate at which the individuals leaving the infected compartment $I$ for quarantine compartment at time $t$. When it is determined that individuals in quarantine have not been infected, they go to recovered class $R, \tau(t)$ denotes this rate. Based on epidemiological meaning, these parameters will be positive.

We assume that $\alpha(t), \gamma(t), \omega(t)$ and $\tau(t)$ are periodical varying parameters. So, for instance, the values of these parameters depend on the season of the year in which we are: spring, summer, autumn and winter. In this example, the system is $P$-periodic with $P=4$.

Considering all the above assumptions, the time evolution of the variables $S(t), I(t), Q(t)$ and $R(t)$ is given by

$$
\left\{\begin{array}{l}
S(t+1)=p S(t)-\alpha(t) S(t) I(t)+\mu(t) N, \\
I(t+1)=(q-\gamma(t)-\omega(t)) I(t)+\alpha(t) S(t) I(t), \\
Q(t+1)=\omega(t) I(t)+(s-\tau(t)) Q(t), \\
R(t+1)=r R(t)+\gamma(t) I(t)+\tau(t) Q(t), \quad t \in \mathcal{Z}^{+} .
\end{array}\right.
$$

We denote this system as the SIQR model. And the feasible region of this model is given by

$$
\Omega=\left\{\left(S(t), I(t), Q(t),(R(t)) \geq 0, / N=S(t)+I(t)+R(t)+Q(t), t \in \mathcal{Z}^{+}\right\} .\right.
$$

For our model to be well posed from the mathematical and epidemiological point of view, it is necessary that $\Omega$ is positively invariant and that there are solutions in $\Omega$ for all non-negative time. Note that, to ensure that this region 
is positively invariant we must impose restrictions on the parameters. This will be taken into account in next sections.

From $N=S(t)+I(t)+R(t)+Q(t)$. Taking into account the relation $\mu(t) N=(1-p) S(t)+(1-q) I(t)+(1-s) Q(t)+(1-r) R(t)$, the first equation of the system (3) is reduced to $S(t+1)=(-q-\alpha(t) S(t)) I(t)-s Q(t)-r R(t)+N$.

\section{Effect of quarantine in the seasonal epidemic model. Equilibrium points. Stability}

In this section, we will analyze the effects of quarantine on the spreading of infectious diseases and study asymptotic stability around the disease-free equilibrium point.

To obtain the equilibrium solutions, we have to take into account the $P$-periodic behavior of the dynamic process. We look for $P$ points $\left\{E_{j}^{*}=\right.$ $\left.\left(S_{j}^{*}, I_{j}^{*}, Q_{j}^{*}, R_{j}^{*}\right), / j=0,1, \ldots, P-1\right\}$, such that if the system reaches that cycle of states, then it stays in it. We need to solve the following system

$$
\left\{\begin{aligned}
S_{j+1}^{*}= & \left(-q-\alpha(j) S_{j}^{*}\right) I_{j}^{*}-s Q_{j}^{*}-r R_{j}^{*}+N, \\
I_{j+1}^{*}= & (q-\gamma(j)-\omega(j)) I_{j}^{*}+\alpha(j) S_{j}^{*} I_{j}^{*} \\
Q *_{j+1}= & \omega(t) I_{j}^{*}+(s-\tau(j)) Q_{j}^{*} \\
R_{j+1}^{*}= & r R_{j}^{*}+\gamma(j) I_{j}^{*}+\tau(j) Q_{j}^{*}, \\
& j=0,1, \ldots, P-1, \text { and } E_{N}^{*}=E_{0}^{*} .
\end{aligned}\right.
$$

By a straightforward manipulation of these equilibrium equations we obtain that there exists a disease-free equilibrium solution given by a unique equilibrium point $E_{f}^{*}=(N, 0,0,0)$ and, moreover, the equilibrium equations (4) also have another solutions. But, it should be noted that for the endemic equilibrium solution to make sense (from the epidemiological point of view) it is necessary that the number of individuals of each compartment be non-negative and, even more, of infected individuals number be positive. In this sense, we obtain a solution of $(4), E_{e}^{*}=\left\{\left(S_{j}^{*}, I_{j}^{*}, Q_{j}^{*}, R_{j}^{*}\right), / j=0,1, \ldots, P-1\right\}$, which is named endemic equilibrium periodic solution.

We summarize the previous comments in the following result.

Theorem 1 System (3) has two equilibrium solutions: The disease-free equilibrium point $E_{f}^{*}$ and the endemic equilibrium periodic solution $E_{e}^{*}$.

Motivated by the previous result, we give two numerical simulations to observe the global behavior of the model around of two equilibrium locations.

Example 1 Consider a population of size $N=300$ in which an outbreak of infectious disease arises showing a 4-periodic behavior corresponding to the 4 seasons. The parameters are given in Table 1.

Depending on the transmission rate, after a sufficiently long time, we observe that the solution of the system tends to one of the equilibrium solutions.

Specifically, in Case (a) with $\alpha=0.005$, a numerical simulation with Mathematica program allow us to know the behavior of the solution. The 
graphs of Figure 1 show that the infected population tend to disappear and the trajectory of the system is asymptotically stable to the disease-free point $E_{f}^{*}=(300,0,0,0)$.

However, in Case (b), with $\alpha=0.008$, solving the equilibrium equations (4) we obtain a endemic equilibrium periodic solution

$$
\begin{gathered}
E_{e}^{*}=\{(199.87,6.68,12.28,81.14),(199.83,6.34,6.83,86.98), \\
(200.44,6.65,8.75,84.14),(200.46,7.01,11.71,80.80)\} .
\end{gathered}
$$

As in the above case, using Mathematica program, we have made the numerical simulation, running iteratively, the difference equations of the system. Then, we have obtained that the solution of the system tends to this endemic equilibrium periodic solution. That is is showed in the Figure 2.

$$
\begin{array}{r}
p=0.95, q=0.75, s=0.93, r=0.9 \\
w(0)=0.6, w(1)=0.7, w(0)=0.8, w(0)=0.7 \\
\gamma(0)=0.8, \gamma(1)=0.6, \gamma(2)=0.5, \gamma(3)=0.7 \\
\tau(0)=0.7, \tau(1)=0.6, \tau(2)=0.5, \tau(3)=0.7
\end{array}
$$

Table 1 Parameters in a 4-periodic epidemic model.

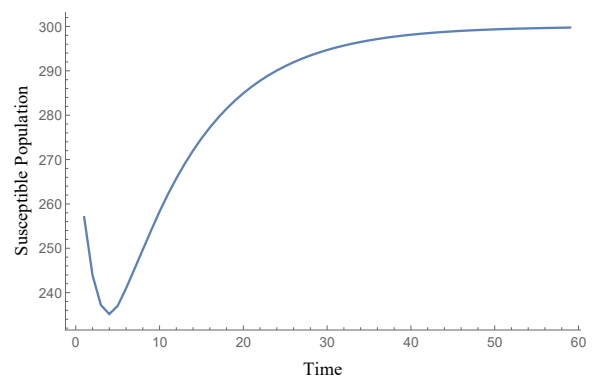

(i) Evolution of population $S$.

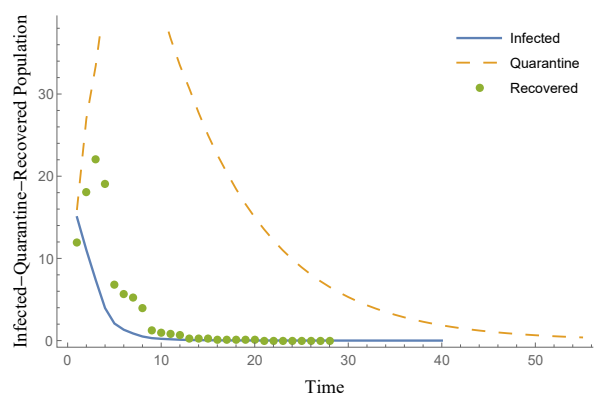

(ii) Evolution of populations $I, Q, R$.

Fig. 1 Case (a). Dynamic of the $N$-periodic model with $\alpha=0.005$.

To determine a threshold condition for which the disease disappears, we need to introduce the reproduction number of the SIQR model. It is called quarantine reproduction number and denoted by $\mathcal{R}_{q}$. This parameter can be obtained by using the next generation method and, as it is known, this is closely related to the analysis of the stability of the model around the diseasefree equilibrium point (Castillo-Chavez et al.(2002); Cao and Zhou (2013) ).

First, we study the global behavior of the model around the disease-free equilibrium point $E_{f}^{*}$. The study of stability of the solution of the system in an environment close to its disease-free equilibrium point we need the eigenvalues 


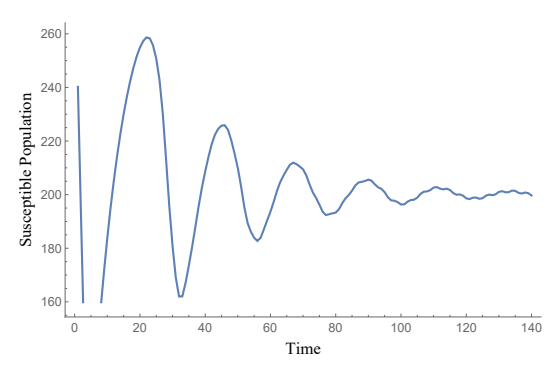

(i) Evolution of population $S$.

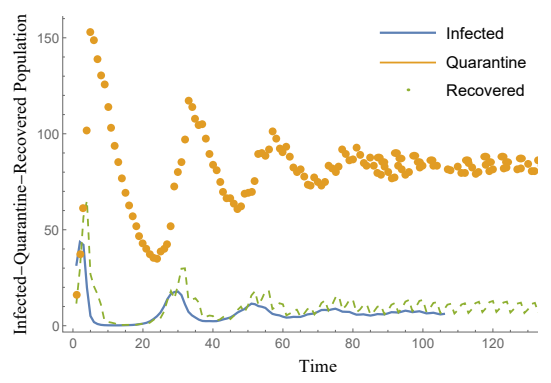

(ii) Evolution of populations $I, Q, R$.

Fig. 2 Case (b). Dynamic of the $N$-periodic model with $\alpha=0.008$.

of the coefficient matrix of the associated linear system near the steady-state solution. Then, the idea is to approximate the nonlinear system by a linear one (around the equilibrium point $E_{f}^{*}$.). To do this, we consider $S(t) I(t) \simeq$ $N I(t)$. Denote $\hat{S}(t)=S(t)-N, \hat{I}(t)=I(t), \hat{Q}(t)=Q(t), \hat{R}(t)=R(t)$ and $x(t)=(\hat{S}(t), \hat{I}(t), \hat{Q}(t), \hat{R}(t))$. Then, we obtain the following linear system

$$
x(t+1)=A(t) x(t), \quad t \in \mathcal{Z}^{+},
$$

where the $P$-periodic matrix $A(t)$ is given by

$$
A(t)=\left(\begin{array}{cccc}
1 & 1-q-\alpha(t) N & 1-s & 1-r \\
0 & q-(\gamma(t)+\omega(t))+\alpha(t) N & 0 & 0 \\
0 & \omega(t) & s-\tau(t) & 0 \\
0 & \gamma(t) & \tau(t) & r
\end{array}\right)
$$

The evolution of the disease state is given by infected individuals, and hence we can reduce this model to the $P$-periodic infected linear model

$$
\hat{I}(t+1)=a(t) \hat{I}(t), \quad t \in \mathcal{Z}^{+},
$$

with the $P$-periodic parameter $a(t)$ given by $a(t)=q+b(t)$ with $b(t)=$ $\alpha(t) N-(\gamma(t)+\omega(t)), \quad t \in J=\{0,1, \cdots, P-1\}$. Note that, the dynamic of the system makes sense if $a(t)>0$, for all $t \in J$.

It is known that if $\rho\left(\Phi_{a}(P, 0)\right)<1$ the system $(7)$ is asymptotically stable. As $a(t)$ is scalar we can obtain the value of $\rho\left(\Phi_{a}(P, 0)\right)=\prod_{i=0}^{P-1} a(i)$ directly by a simple calculation, but it is a fairly complex expression that involves products with the parameters.

We are interested in knowing information about the behavior of the model based on the parameters that appear in it. So, we use the concept of basic reproduction number for $P$-periodic system given Bacaër and Ait (2012) . So, to obtain the quarantine reproduction number $\mathcal{R}_{q}^{e}$ we need the ICAS formulation for the periodic system (7), given by

$$
z(t+1)=A_{e} z(t), \quad A_{e}=T_{e}+F_{e}, \quad t \geq 0
$$


where

$$
T_{e}=\left(\begin{array}{ccccc}
0 & 0 & \cdots & 0 & q \\
q & 0 & \cdots & 0 & 0 \\
0 & q & \cdots & 0 & 0 \\
\vdots & \vdots & \ddots & \vdots & \vdots \\
0 & 0 & \cdots & q & 0
\end{array}\right) \text { and } F_{e}=\left(\begin{array}{ccccc}
0 & 0 & \cdots & 0 & b(0) \\
b(1) & 0 & \cdots & 0 & 0 \\
0 & b(2) & 0 & \cdots & 0 \\
\vdots & \vdots & \ddots & \vdots & \vdots \\
0 & 0 & \cdots & b(P-1) & 0
\end{array}\right)
$$

For defining the quarantine reproduction number $\mathcal{R}_{q}^{e}$ we use the next generation method, that is, $\mathcal{R}_{q}^{e}=\rho\left(F_{e}\left(I-T_{e}\right)^{-1}\right)$, if $\mathcal{R}_{q}^{e}<1$ the disease disappears and no extra control measures are required in order to maintain a disease-free population, while for $\mathcal{R}_{q}^{e} \geq 1$, the epidemic can possibly establish in the population and increase rapidly, Castillo-Chavez et al. (2002). By definition of $T_{e}$ and $F_{e}$ the matrix $F_{e}\left(I-T_{e}\right)^{-1}=D_{e} \Lambda_{e}$ where

$$
D_{e}=\operatorname{diag}(b(0), \ldots, b(P-1))
$$

and $\Lambda_{e}$ is a circulant matrix, Davis (1979), defined by

$$
\left(\frac{q^{P-1}}{1-q^{P}}, \ldots, \frac{q}{1-q^{P}}, \frac{1}{1-q^{P}}\right) .
$$

And using $0<q<1$ it follows that $\Lambda_{e}$ is a non-negative matrix.

Lemma 1 The spectral radius of the circulant matrix $\Lambda_{e}$ is $\rho\left(\Lambda_{e}\right)=\frac{1}{1-q}>1$.

Proof The eigenvalues of matrix $\Lambda_{e}$ are $\lambda_{j}=\sum_{k=0}^{P-1} q^{P-1-k} w_{j}^{k}$, with $w_{j}=$ $\operatorname{Exp}\left(\frac{2 \pi j}{P} \mathbf{i}\right), j=0,1, \ldots, P-1$. From definition of $w_{j}$ it follows $\left|w_{j}\right|=1, \forall j$. Since,

$$
\left|\lambda_{j}\right| \leq \frac{1}{1-q^{P}} \sum_{k=0}^{P-1} q^{P-1-k}\left|w_{j}^{k}\right|=\frac{1}{1-q^{P}} \sum_{k=0}^{P-1} q^{P-1-k}=\lambda_{0} \geq 0
$$

we have $\rho\left(\Lambda_{e}\right)=\lambda_{0}$. On the other hand

$$
\rho\left(\Lambda_{e}\right)=\frac{\sum_{k=0}^{P-1} q^{P-1-k}}{1-q^{P}} \frac{1-q}{1-q}=\frac{1-q^{P}}{\left(1-q^{P}\right)(1-q)}=\frac{1}{1-q} .
$$

From $0<q<1$ it follows $\rho\left(\Lambda_{e}\right)>1$.

Now, we focus our attention on parameter $b(t)$. Note that, the definition of $b(t)$ is closely related to the recovery and quarantine rates. Therefore, $b(t)$ will be smaller when some of these rates increase. As $a(t)$ should be positive for all $t$ the definition domain of our process, then $\alpha(t) N+q>\gamma(t)+\omega(t)$. Thus, in this SIQR model, the rate of isolated individuals at each step, $\omega(t)$, will be decisive in the evolution of the disease. Moreover, if it is possible to choose $\omega(t)>0$ such that $\alpha(t) N>\gamma(t)+\omega(t)$ we have $a(t)>0$ with $b(t)>0$. 
Motivate by this, our goal of analyzing the behavior of the epidemiological process on quarantine approach is centered in this parameter. Denoting by $b_{u}=\max \{b(t), t \in J\}$ and by $b_{l}=\min \{b(t), t \in J\}$ with $J=\{0,1, \ldots, P-1\}$, we have the following results.

Lemma 2 Consider the matrices $D_{e}$ and $\Lambda_{e}$ and the previously defined $b_{u}, b_{l}$ with $b_{l}>0$. Then

(i) $\rho\left(D_{e} \Lambda_{e}\right) \leq b_{u} \rho\left(\Lambda_{e}\right)$ and $\rho\left(D_{e} \Lambda_{e}\right) \geq b_{l} \rho\left(\Lambda_{e}\right)$.

(ii) If $q^{P-1}(1+q) \geq 1$ then $\rho\left(D_{e} \Lambda_{e}\right) \geq \rho\left(D_{e}\right)$.

Proof (i) As $\Lambda_{e}, D_{e}$, and $D_{e} \Lambda_{e}$, are non-negative matrices and $b_{l}>0$, we have $D_{e} \Lambda_{e} \leq b_{u} \Lambda_{e}$ and $D_{e} \Lambda_{e} \geq b_{l} \Lambda_{e}$. From here and Corollary 1.5 in Berman and Plemmons (1994), it follows directly that $\rho\left(D_{e} \Lambda_{e}\right) \leq b_{u} \rho\left(\Lambda_{e}\right)$, $\rho\left(D_{e} \Lambda_{e}\right) \geq b_{l} \rho\left(\Lambda_{e}\right)$.

(ii) If $q^{P-1}(1+q) \geq 1$ we have $D_{e} \Lambda_{e} \geq D_{e}$ then $\rho\left(D_{e} \Lambda_{e}\right) \geq \rho\left(D_{e}\right)$.

Theorem 2 Consider the matrices $D_{e}$ and $\Lambda_{e}$ and the previously defined $b_{u}, b_{l}$ with $b_{l}>0$. Then

(i) If $b_{u}<1-q$ then the disease free equilibrium $E_{f}$ of the SIQR model is asymptotically stable in the region $\Omega$.

(ii) If $b_{l} \geq 1-q$ then the disease free equilibrium $E_{f}$ of the SIQR model is not asymptotically stable in the region $\Omega$.

(iii) If $q^{P-1}(1+q) \geq 1$ and there exists $i_{0} \in J$ such that $\alpha\left(i_{0}\right)>\frac{1+\gamma\left(i_{0}\right)+\omega\left(i_{0}\right)}{N}$ then the disease free equilibrium $E_{f}$ of the SIQR model is not asymptotically stable in the region $\Omega$.

Proof By definition, $\mathcal{R}_{q}^{e}=\rho\left(F_{e}\left(I-T_{e}\right)^{-1}\right)=\rho\left(D_{e} \Lambda_{e}\right)$. (i)-(ii) From Lemma $2(i)$ and $\rho\left(\Lambda_{e}\right)=\frac{1}{1-q}$, we have

$$
\frac{b_{l}}{1-q} \leq \mathcal{R}_{q}^{e} \leq \frac{b_{u}}{1-q}
$$

Hence, if $b_{u}<1-q$ then $\mathcal{R}_{q}^{e}<1$ and the disease free equilibrium $E_{f}$ of the system (4)-(??) is asymptotically stable in the region $\Omega$. And, if $b_{l} \geq 1-q$ then $\mathcal{R}_{q}^{e} \geq 1$ and the disease free equilibrium $E_{f}$ of the system (4)-(??) is not asymptotically stable in the region $\Omega$.

(iii) From Lemma 2 (ii), $\mathcal{R}_{q}^{e} \geq \rho\left(D_{e}\right)$ and $\rho\left(D_{e}\right)$ is the maximum modulus of $b(t)=\alpha(t) N-(\gamma(t)+\omega(t)), t \in J$. If there exists $i_{0} \in J$ such that $1+\gamma\left(i_{0}+\omega\left(i_{0}\right)<\alpha\left(i_{0}\right) N\right.$ then $b\left(i_{0}\right)>1$ and $\rho\left(D_{e}\right)>1$. So, $\mathcal{R}_{q}^{e}>1$ and the disease free equilibrium $E_{f}$ is not asymptotically stable.

Remark 1 In Theorem 2 we have given some conditions on the parameters of the model which depend on parameter $\omega(t)$.

In $(i)$ the condition confirms that the quarantined individuals are effective in leading to the extinction of the disease. It is shown that if we get a quarantine rate $\omega(t)$ so that $b_{u}$ is less than the mortality rate of infected individuals then the disease is eradicated. 
In sections $(\boldsymbol{i i})$ and (iii) the conditions established determine the inefficiency of the quarantine as a control strategy for our model. In particular, (iii) shows that when a disease with a small mortality rate and high virulence occurs, it is not effective to apply quarantine as a control action.

The evolution of the individuals of each compartment is given in Figure 3

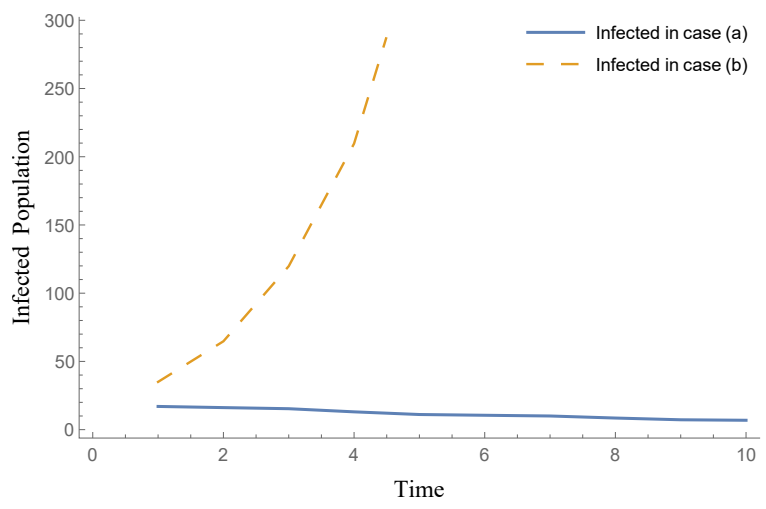

Fig. 3 Dynamic of the $N$-periodic model with $\alpha=0.008$.

The following result, which is given without proof, is a direct consequence of the previous theorem.

Corollary 1 If $b(t)=b, t \in J$, then

$$
\mathcal{R}_{q}^{e}=\frac{b}{1-q}
$$

Moreover, if $0<b<1-q$ the disease free equilibrium $E_{f}$ of the SIQR model is locally asymptotically stable in the region $\Omega$; otherwise $E_{f}$ is unstable.

Remark 2 From definition $b(t)$ as the sum of several parameters, the condition $b(t)=b, t \in J$, does not imply that the periodicity in the model is lost. Also, if we can determine the quarantine rate in each step, the conditions of the previous result could be achieved. Thus, can determine the rate of individuals that must be quarantined by $\omega(t)=\alpha(t) N-(\gamma(t)+b) \geq 0$, for each $t \in J$.

In the next result, we give some conditions to assure the stability of the periodic system (7) in the case that some of the $b(t)$ are null.

Theorem 3 Let $b(j)=\alpha(j) N-(\gamma(j)+\omega(j)) j \in J$ be.

(a) If there exists a unique $i_{0} \in J$ such that $b\left(i_{0}\right) \neq 0$ then

(i) the basic reproduction number

$$
\mathcal{R}_{q}^{e}=\mid\left(b\left(i_{0}\right) \mid \frac{q^{P-1}}{1-q^{P}} .\right.
$$


(ii) if $\alpha\left(i_{0}\right)<\frac{1-q^{P}}{q^{P-1} N}$ the disease disappears.

(b) If there exists $i_{1}, i_{2} \in J$ such that $b\left(i_{1}\right)=b\left(i_{2}\right)=b$ and $b(j)=0$ for all $j \in J, j \neq i_{1}, i_{2}$ then

(i) the basic reproduction number

$$
\mathcal{R}_{0}^{e}=\frac{|b| q^{\frac{P}{2}}}{\left(1-q^{\frac{P}{2}}\right) q}
$$

(ii) if $\alpha(j)<\frac{\left(1-q^{\frac{P}{2}}\right) q}{N q^{\frac{P}{2}}}$, for $j \in\left\{i_{1}, i_{2}\right\}$, the disease disappears.

Proof (a) Without loss of generality we assume that $i_{0}=0$.

(i) The eigenvalues of $F_{e}\left(I-T_{e}\right)^{-1}$ are $\lambda_{j}=0, j=1, \ldots, P-1$ and $\lambda_{0}=(\alpha(0) N-(\gamma(0)+\omega(0))) \frac{q^{P-1}}{1-q^{P}}$. Then the spectral radius is $\left|\lambda_{0}\right|$ and by definition of the basic reproduction number, $\mathcal{R}_{0}^{e}=\left|\lambda_{0}\right|$.

(ii) If $\alpha(0)<\frac{1-q^{P}}{q^{P-1} N}$, using (i) we have $\mathcal{R}_{q}^{e}=|\alpha(0) N-(\gamma(0)+\omega(0))| \frac{q^{P-1}}{1-q^{P}}$. Since $\gamma(0)+\omega(0)>0$, we have

$$
(\alpha(0) N-\gamma(0)-\omega(0)) \frac{q^{P-1}}{1-q^{P}}<\alpha(0) N \frac{q^{P-1}}{1-q^{P}}<1 .
$$

Then, the system is asymptotically stable and the disease disappears.

(b) Without loss of generality we assume that $i_{1}=0$ and $i_{2}=1$. By hypothesis, $b(0)=b(1)=b$.

(i) The eigenvalues of $F_{e}\left(I-T_{e}\right)^{-1}$ are $\lambda_{j}=0, j \in J-\left\{i_{1}, i_{2}\right\}$, and $\lambda_{0}=\frac{b q^{\frac{P}{2}}}{q\left(1-q^{\frac{P}{2}}\right)}$ and $\lambda_{1}=-\frac{b q^{\frac{P}{2}}}{q\left(1+q^{\frac{P}{2}}\right)}$. From $q<1$, we have $0<$ $1-q^{\frac{P}{2}}<1+q^{\frac{P}{2}}$, then $\left|\lambda_{0}\right|>\left|\lambda_{1}\right|$.Then by definition of the basic reproduction number, $\mathcal{R}_{0}^{e}=\left|\lambda_{0}\right|$.

(ii) If $\alpha(j)<\frac{\left(1-q^{\frac{P}{2}}\right) q}{N q^{\frac{P}{2}}}$ for $j \in\left\{i_{1}, i_{2}\right\}$, using (i) and taking into account that $\gamma(j)+\omega(j)>0$, we have

$$
\mathcal{R}_{q}^{e}=\mid\left(\alpha(j) N-(\gamma(j)+\omega(j)) \mid \frac{1}{\left(1-q^{\frac{P}{2}}\right) q^{1-\frac{P}{2}}}<\frac{\alpha(j) N}{\left(1-q^{\frac{P}{2}}\right) q^{1-\frac{P}{2}}}<1 .\right.
$$

Then, the system is asymptotically stable and the disease disappears.

In the previous result, we have obtained some conditions that allow us to guarantee the efficacy of the quarantine to eradicate the disease. The condition that any of the $b(t)$ be null can be obtained whenever it is possible to determine the rate of individuals that must be quarantined in each of the stations that we have considered in our model. If this happens, we can obtain the rate of individuals that must be quarantined using $\omega(t)=\alpha(t) N-\gamma(t) \geq 0, t \in J$. 


\section{Conclusions}

This paper had discussed the effect of quarantine on the spreading of a seasonal disease. For this, we have introduced a quarantined compartment in a susceptible-infected-recovered (SIR) seasonal model and the quarantined rate is included in the model as a periodic function. It is showed that the proposed SIQR model has a unique disease equilibrium point $E_{f}^{*}$ in the feasible region $\Omega$, which under certain conditions, turns out to be asymptotically stable and a unique endemic equilibrium $E_{e}^{*}$ in that region. The quarantine reproduction number $\mathcal{R}_{q}^{e}$ is defined by the next generation method. From this indicator $\mathcal{R}_{q}^{e}$, some conditions are given on the parameters of the model to analyze the effectiveness or not of the use of quarantine as a control action to eradicate the disease.

Acknowledgements This work is supported by Spanish Grant MTM2013-43678-P.

\section{References}

1. L. Allen, P. van den Driessche, The basic reproduction number in some discrete-time epidemic models, J. Difference Equ. Appl., 1-19, (2008).

2. N. Bacaër, E.H.Ait Dads, On the biological interpretation of a definition for the parameter $R_{0}$ in periodic population models, Journal Mathematical Biology, 601-621, (2012).

3. A. Berman, R.J. Plemmons, non-negative Matrices in the Mathematical Sciences, SIAM, Philadelphia (1994).

4. C. Castillo-Chavez, C. W. Castillo-Garsow, A. A. Yakubu, Mahematical models of isolation and quarantine, JAMA, 290 2876- 2877 (2003).

5. B. Cantó, C. Coll, E. Sánchez, A study on vaccination models for a seasonal epidemic process, Applied Mathematics and Computation, 243 152- 160 (2014).

6. C. Castillo-Chavez, Z. Feng, W. Huang, On the computation of $R_{0}$ and its role on global stability, Institute for Mathematics and Its Applications, 125 229- 251 (2002).

7. S. Cauchemez, A.J. Valleron, P.Y. Boelle, A. Flahault, N.M. Ferguson, Estimating the impact of school closure on inluenza transmission from Sentinel data, Nature, 452750 $754,(2008)$.

8. H. Cao, Y. Zhou, The basic reproduction number of discrete SIR and SEIS models with periodic parameters, Discrete and continuous dynamical systems series B, 18 (1) 37- 56 (2013).

9. B.J. Coburn, B. G. Wagner, S. Blower, Modeling influenza epidemics and pandemics: insights into the future of swine $\mathrm{flu}$, BMC Medicine, 7:30 http://www.biomedcentral.com/1741-7015/7/30, (2009).

10. J.M. Cushing, A.S. Ackleh, A net reproductive number for periodic matrix models. J. Biol. Dyn. , 6(2), 166-188 (2012).

11. P. Davis, Circulant Matrices, Wiley, New York (1979).

12. J.M. Epstein, D.M. Goedecke, F. Yu, R.J. Morris, D.K. Wagener, G.V. Bobashev, Controlling pandemic flu: the value of international air travel restrictions, PLOS ONE, 452:e401 (2007).

13. L. Fumanelli, M. Ajelli, S. Merler, N. M. Ferguson, S. Cauchemez, ModelBased Comprehensive Analysis of School Closure Policies for Mitigating Influenza Epidemics and Pandemics, PLOS Computational Biology, (2016), https:// doi.org/10.1371/journal.pcbi.1004681 .

14. H. Hetcote, M. Zhien, L. Shengbing, Effects of quarantine in six endemic models for infectious diseases. Mathematical Biosciences, 180, 141-160 (2002).

15. C.K. Li, H. Schneider, Applications of Perron-Frobenius theory to population dynamics, Journal Mathematical Biology, 44, 450-462 (2002). 
16. R.A. Meyer, C.S. Burrus, A unified analysis of multirate and periodically time-varying digital filters, IEEE Trans. Circuits Systems, 22(3), 162-168 (1975).

17. A. M. Mubayi, C.K. Zaleta, M. Martcheva, C. Castillo-Chavez, A cost-based comparison of quarantine strategies for new emerging diseases, Mathematical Biosciences and Engieneering, 7(3), 687-717 (2010).

18. B. Park, E.I. Verriest, Canonical forms on discrete linear periodically time-varying system and a control application, in: Procc. 28th IEEE Conf. on Decision and Control, Tampa, 1220-1225 (1989).

19. L. Stone, R. Olinky, A. Huppert, Seasonal dynamics of recurrent epidemics, Nature 446 533-536, (2007). 